Phytopath. Z., 101, 281-297 (1981)

(C) 1981 Verlag Paul Parey, Berlin und Hamburg

ISSN 0031-9481 / InterCode: PHYZA3

Aus dem Institut für Pflanzenpathologie und Pflanzenschutz der Georg-August-Universität Göttingen

\title{
Der Einfluß der Spaltöffnungsweite und des Blattalters auf den Infektionserfolg von Cercospora beticola bei Zuckerrüben (Beta vulgaris L.) unterschiedlicher Anfälligkeit
}

\author{
Von \\ F. Feindt, K. Mendgen und R. Heitefuss
}

Mit 11 Abbildungen

Eingegangen am 3. Oktober 1980

Für die Wirt-Parasit-Kombination Cercospora beticola-Zuckerrübe (Beta vulgaris) liegen zahlreiche physiologische Untersuchungen vor, welche die Resistenz der Rübe gegen Cercospora beticola an Hand der Bildung fungitoxischer Substanzen im Gewebe der resistenten Pflanze zu erklären versuchen (z. B. Harrison et al. 1970, Brillova et al. 1973, Hecker et al. 1975, JohnSON et al. 1976, MARTin 1977). Der Pilz wächst zunächst auf der Blattoberfläche, er kann nur durch die Spaltöffnungen in das Wirtsgewebe eindringen. Viele cytologische Arbeiten befassen sich daher mit dem Einfluß der Spaltöffnungen auf die Resistenz der Rübe (Pool und McKay 1916, Canova 1959, Solel et al. 1971, Ruppel 1972, Rathaiah 1977). Widersprüchlich blieb bisher, ob Cercospora beticola durch geschlossene Spaltöffnungen eindringen kann und ob hier ein Zusammenhang mit der Resistenz der Pflanze besteht. Die vorliegende Arbeit versucht mit Hilfe von licht- und elektronenmikroskopischen Untersuchungen, unterstützt durch physiologische Versuche, erneut diesem Fragenkomplex nachzugehen.

\section{Material und Methoden}

\section{Pflanzenmaterial}

Als anfälliges Pflanzenmaterial wurde eine hochanfällige „N-Rübenlinie" der KleinStammzuch einer diploiden "CR-Linie" (T 48) der KWS mit einem gut ausgeprägten Rewanzlebener Saatzucht (KWS) verwendet. Das resistente Material stamme aus einer Familiensistenzgrad. Die Anzucht der Pflanzen erfolgte nach dreimaligem. Umtopfen ab der fünften 
Woche der Aussaat in $18 \times 18 \mathrm{~cm}$ Plastiktöpfen in einem Kompost-Einheitserde Typ T-Gemisch von $1: 1$ bei einem Tag/Nacht-Rhythmus von $20 / 4 \mathrm{~h}$, einer Anzuchttemperatur von $23 / 16^{\circ} \mathrm{C}$ $\pm 1^{\circ} \mathrm{C}$, einer relativen Luftfeuchte von $70 / 90 \%$ und einer Lichtintensität von 22000 bis 24000 L.ux in einer Weiß-Klimakammer.

\section{Pilzherkunft und -kultivierung}

In allen Versuchen wurde Cercospora beticola (Herkunft 'Isolat Einbeck') verwendet. Nach zwei- bis dreimonatiger Kultivierung auf Nährmedium wurde jeweils eine Wirtspassage $z w i s c h e n g e s c h a l t e t, u m$ eine Virulenzminderung zu vermeiden. Die Kultivierung erfolgte in Anlehnung an LA (1963) auf Gemüsesaftagar (50\% V-8, 2 bis $3 \%$ Agar) pH 6,8, die Inkubation bei Raumtemperatur unter indirektem Tageslichteinfluß. Nach drei bis vier Tagen Inkubation ließ sich von einem gleichmäßigen Pilzrasen reichlich einheitliches Konidienmaterial mit $0,1 \%$ iger Lubrollösung und einem Haarpinsel abwaschen.

\section{Infektion der Pflanzen und Inkubation}

Die Blätter der Versuchspflanzen wurden einzeln aus etwa $40 \mathrm{~cm}$ Entfernung mit Hilfe einer Düse bei einem Druck von etwa $1 \mathrm{kp} / \mathrm{cm}^{2}$ gleichmäßig mit einer vorher durch ein grobes Nesseltuch gegebenen Konidiensuspension besprüht. Für die lichtmikroskopischen und rasterelektronenmikroskopischen Untersuchungen wurde eine Konidiendichte von etwa drei bis fünf Konidien je $\mathrm{mm}^{2}$ Blattfläche angestrebt, während für die Untersuchungen mit dem Transmissions-EM die Infektionsdichte um das Zehnfache erhöht wurde. In Anlehnung an Canova (1959) und BleIholder (1971) erfolgte die Inkubation in Feuchtkammern, die in einer Gewächshauskabine aufgestellt waren. Als Lichtquelle diente das normale Tageslicht. Zur Erhöhung der Luftfeuchte war der Boden der Feuchtkammer mit einer $5 \mathrm{~cm}$ hohen, angefeuchteten Torfmullage bedeckt. Während der Inkubation wurde eine Wassersättigung der Luft mit einem intervallgesteuerten Defensor erreicht und mit einem Thermohygrographen kontrolliert. Während der Inkubationszeit von 84 bis 96 Stunden wurde die relative Luftfeuchte jeweils nach 24, 48 und 72 Stunden für 3 Stunden von $100 \%$ r.L. auf 75 bis $80 \%$ r.L. herabgesetzt.

Nach der Inkubation wurden die Pflanzen in der Gewächshauskabine oder in der Klimakammer bei 70 bis $80 \%$ r.L., einem 16-Stunden-Tag und den unter 1. genannten Lichtintensitäten zur weiteren Symptomausprägung gehalten.

\section{Ermittlung des stomatären Diffusionswiderstandes}

Die Messungen des stomatären Diffusionswiderstandes der Blätter erfolgten in der Klimakammer jeweils 2 Stunden nach Herabsetzung der Lichtintensität auf 1000 Lux mittels eines in der Werkstatt des Institutes hergestellten Diffusionsporometers (in Anlehnung an Kanemasu et al. 1969).

Der Druckabfall wurde im Bereich von 100 bis 60 Torr an einem "Manuell Autotest“Manometer eines Blutdruckmeßgerätes beobachtet. Mittels einer Stoppuhr wurde im Zeitbereich von 2 bis $40 \mathrm{sec}$ die Zeitspanne des Druckabfalls ermittelt und auf Sekunden je cm Hg umgerechnet.

\section{Applikation von Abscisinsäure (ABS)}

Um den Einfluß des Offnungsgrades der Spaltöffnungen zu untersuchen, wurden die jeweils zu behandelnden Blätter morgens und abends an vier aufeinanderfolgenden Tagen auf ihrer Unterseite mit einer Lösung von $40 \mathrm{mg} / \mathrm{l} \mathrm{ABS}$ in dest. Wasser besprüht.

\section{Ermittlung des Pilzbefalls und der Pilzentwicklung}

\subsection{Befallsgrad}

Der Befallsgrad wurde in Anlehnung an die "KWS-Cercospora-Bonitierungstafel“ geschätzt. Da sich der Cercospora-Befallsgrad der Bonitierungstafel im Freiland auf die ganze Pflanze bezieht, in den Gewächshausversuchen aber nur die inokulierte Blattfläche einzelner 
13lätter berücksichtigt werden konnte, mußten die Befallszahlen in folgender Weise modifiziert xerden:

$0=0$ bis $10 \%, 1=10$ bis $20 \%, 2=20$ bis $30 \%, \ldots 9=90$ bis $100 \%$

der inokulierten Blattflächen nekrotisiert.

\subsection{Konidienkeimung und Hyphenwachstum}

Die Konidienkeimung wurde direkr an Hand der Anzahl Primärhyphen am 2.d.p.i. ermittelt. Das Hyphenwachstum wurde nicht über die Hyphenlänge, sondern indirekt über die Anzahl gebildeter Sekundärhyphen am 5.d.p.i. bestimmt. Als Primärhyphe wurde eine Hyphe bezeichnet, wcnn sie direkt aus einer Konidienzelle hervorgegangen war, als Sekundärhyphen diejenigen, die von Primärhyphen abzweigten. Die Anzahl der Primär- und Sekundärhyphen wurde auf den Durdischnitt der ausgewerteten Konidien bezogen. Je Blattprobe wurden zehn Konidicn ausgewertet.

\subsection{Stomakontakt und Penetrationsrate}

Als Stomakontakt vurde jeder Kontakt einer Hyphe mit einer Schließzelle am 5.d.p.i. bezeichnet. Als Penetrationserfolg einer Konidie wurde gewertet, wenn eine ihrer Hyphen mit der Spitze die untere Sdlließzellenleiste einer Spaltöffnung erreicht hatte. Dieses Kriterium konnte im Lichtmikroskop bei 250facher Vergrößerung an aufgehellten Blattproben relativ leicht und gut beurteilt werden.

\section{Präparationsmethoden für die Lichtmikroskopie}

Die Konidienkeimung wurde in Anlehnung an SAMpson (1961) und Zelitch (1961) an Negativabdrücken ermittelt. Die Nagellackmethode nach Anfärben der Hyphen mit $0,1 \%$ Säurefuchsin erwies sich dazu als am besten geeignet.

Zur Ermittlung der Penetrationsrate wurden die Blattproben nach GerLACH (1969) in Lactophenol aufgehellt und die Pilzhyphen mit Anilinblau angefärbt.

\section{Präparationstechnik für die Rasterelektronenmikroskopie}

Für die Untersuchungen im Raster-EM wurden die Proben in Anlehnung an Boyde et al. (1969) und D $\mathrm{y}$ et al. (1973) in folgender Weise aufgearbeitet:

Blattproben wurden mit einem Korkbohrer $(\phi 10 \mathrm{~mm})$ unmittelbar vor der Präparation cutnommen, mit „Uhu“ auf einen Objekthalter geklebt, mit Leitsilber umrandet und in einer Balzers-Hochvakuum-Bedampfungsanlage BAE 120 Typ 250 zunächst mit Kohle bis zur Blaufärbung des Präparates $(200 \bar{A})$ bedampft. Danach erfolgte eine Schrägbedampfung mit etwa 80 bis $100 \AA$ Gold mit einem Winkel von $30^{\circ}$. Anschließend wurde die Probe in ein Cambridge "Stereoscan“ Typ 96113, Mark 2A überführt und bei einer Spannung von $30 \mathrm{kV}$ ausgewertet.

\section{Statistische Auswertung}

Die statistische Auswertung erfolgte mit Tischrechnern der Firma Hewlett-Packard, Modell HP-65 und Modell HP-95. Die Daten wurden varianzanalytisch verrechnet und die Unterschiede zwischen den anfälligen und resistenten Rübenlinien mit dem $t$-Test gesichert. Wenn keine Signifikanz vorlag, wurden die Werte der beiden Rübenlinien zusammengefaßt und eine Regressions- und Korrelationsrechnung durchgeführt und der Einfluß des Blattalters bewertet. Die Prüfung auf Linearität der Regression erfolgte mit dem F-Test. Die Versuche über den Einfluß des Pflanzenalters sind mit den gleitenden Mittelwerten nach RENNer dargestellt. 


\section{Ergebnisse}

1. Das äußere Erscheinungsbild anfälliger und resistenter Rüben nach Infektion mit $\boldsymbol{C}$. beticola

Das Auftreten der ersten Krankheitssymptome wird makroskopisch zuerst als Aufhellung des Blattgewebes und Einsenken der Epidermis im Bereich der späteren Nekrose sichtbar. Dieses im Freiland zu beobachtende Symptombild konnte bei unseren Gewächshausversuchen nach einer Inkubationszeit von acht bis zwölf Tagen beobachtet werden. Nach weiteren zwei bis drei Tagen verfärbte sich der im allgemeinen 2 bis $4 \mathrm{~mm}$ große Blattfleck von hellgrün nach hellbraun bis dunkelbraun. Die Anzahl der Blattflecken nahm stark zu. Die gesamten Symptome entwickelten sich unter unseren Inkubationsbedingungen innerhalb einer Woche. Eine Beurteilung des Befallsgrades konnte zwischen dem 15. und 20. Tag nach Inokulation vorgenommen werden.

Bei den anfälligen Rüben wurde ein gleichbleibend hoher Befallsgrad mit zunehmendem Pflanzenalter beobachtet. Bei den resistenten Rüben zeigte sich in den Gewächshaus- und Klimakammerversuchen erst mit zunehmendem Alter (sechs bis sieben Wochen) ein verringerter Befallsgrad (Abb. 1a). Dies bedeutet für Resistenzprüfungen im Gewächshaus, daß die resistente Rübenlinie erst nach sieben bis acht Wochen Kultur durch einen geringeren Befallsgrad von der anfälligen Rübenlinie ausreichend genau unterschieden werden kann.

Bei einer Beurteilung der Symptomentwicklung an einzelnen Blättern acht Wochen alter Pflanzen zeigte sich eine deutliche Abhängigkeit des Befallsgrades vom Alter der Blätter (Abb. 1b).

Unter Berücksichtigung des Blattgradienten lag die anfällige Rübenlinie um etwa zwei Befallsgrade höher als die resistente Linie. Innerhalb einer Linie schwankte jedoch der Befallsgrad zwischen den älteren Blättern (erstes Blatt-

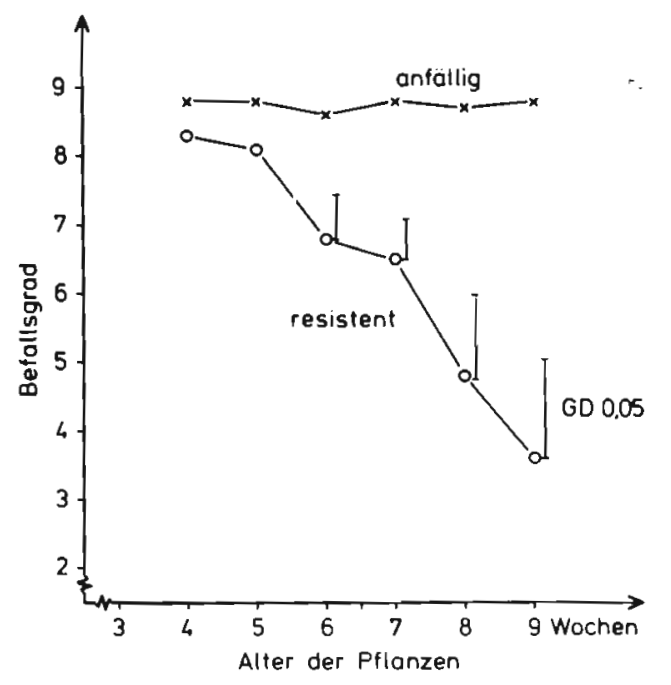

Abb. 1a. Cercospora-Befallsgrad in Abhängigkeir vorm Alter der Pflanzen, 18 d.p.i 
Abb. 1b. Einfluß des Blattalters auf den Befallsgrad bei acht Wochen alten Pflanzen, 18 d.p.i.

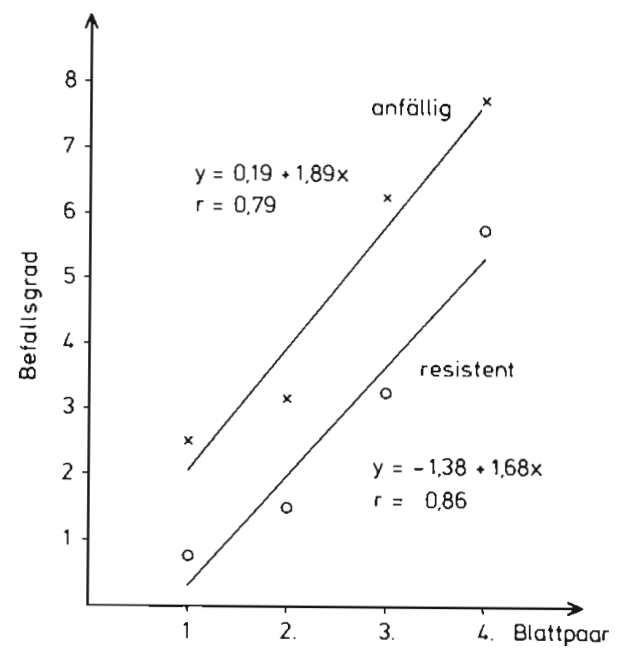

paar) und den jüngeren Blättern (viertes Blattpaar) einer Pflanze um fünf Befallsgrade; bei der anfälligen Linie zwischen zwei und sieben und bei den resistenten Rüben zwischen null und fünf. Daraus folgt, daß jüngere Blätter resistenter Rüben den gleichen Befallsgrad wie ältere Blätter anfälliger Pflanzen erreichen können. Bei Cercospora-Resistenzprüfungen von Zuckerrüben im Gewächshaus sollte daher das unterschiedliche Alter der Blätter berücksichtigt werden.

\section{Konidienkeimung, Hyphenwachstum und Penetration der Hyphen, 2 bis 5 d.p.i.}

Die Keimung der Konidien wird von äußeren Faktoren wie Temperatur und Feuchrigkeit deutlich beeinflußt.

Bei 100 bis $97 \%$ r.L. findet volle, bei $96 \%$ r.L. nur partielle Auskeimung der Konidien statt. Bei $95 \%$ r.L. ist die Keimung eingestellt (FrandsEN 1956).

Bei einem Temperaturoptimum von 23 bis $27^{\circ} \mathrm{C}$ keimt die Konidie innerhalb von 1 bis 2 h zunächst an ihrem basalen und apikalen Ende aus. Später entstehen auch an den mittleren Konidienzellen Keimhyphen.

Der weitere Wachstumsverlauf der Hyphen auf der Blattfläche scheint in den meisten Fällen ungerichtet zu sein. Wir konnten häufig beobachten, daß Hyphen dicht an Spaltöffnungen vorbei (siehe Abb. 2) oder auch direkt darüber hinweg gewachsen waren (siehe Abb. 3), ohne jegliche Anzeichen, in diese einzudringen. Nach längerer Inkubationszeit konnte dann allerdings beobachtet werden, wie Sekundärhyphen von den an Stomata vorbei - bzw. darüber hinweggewachsenen Primärhyphen abzweigten und in Richtung Schließzellen (Abb. 4) oder Atemhöhle (Abb. 5) tendierten. Die Penetration der Hyphen durch die Spaltöffnung erfolgt in der Regel mit einer sogenannten "Infektionshyphe" (Canova 1959), die sich in Form und Struktur deutlich vom übrigen Hyphenabschnitt unterscheidet (Abb.6). Diese Infektionshyphe ist anscheinend identisch mit der von Solel und MrNz (1971) 

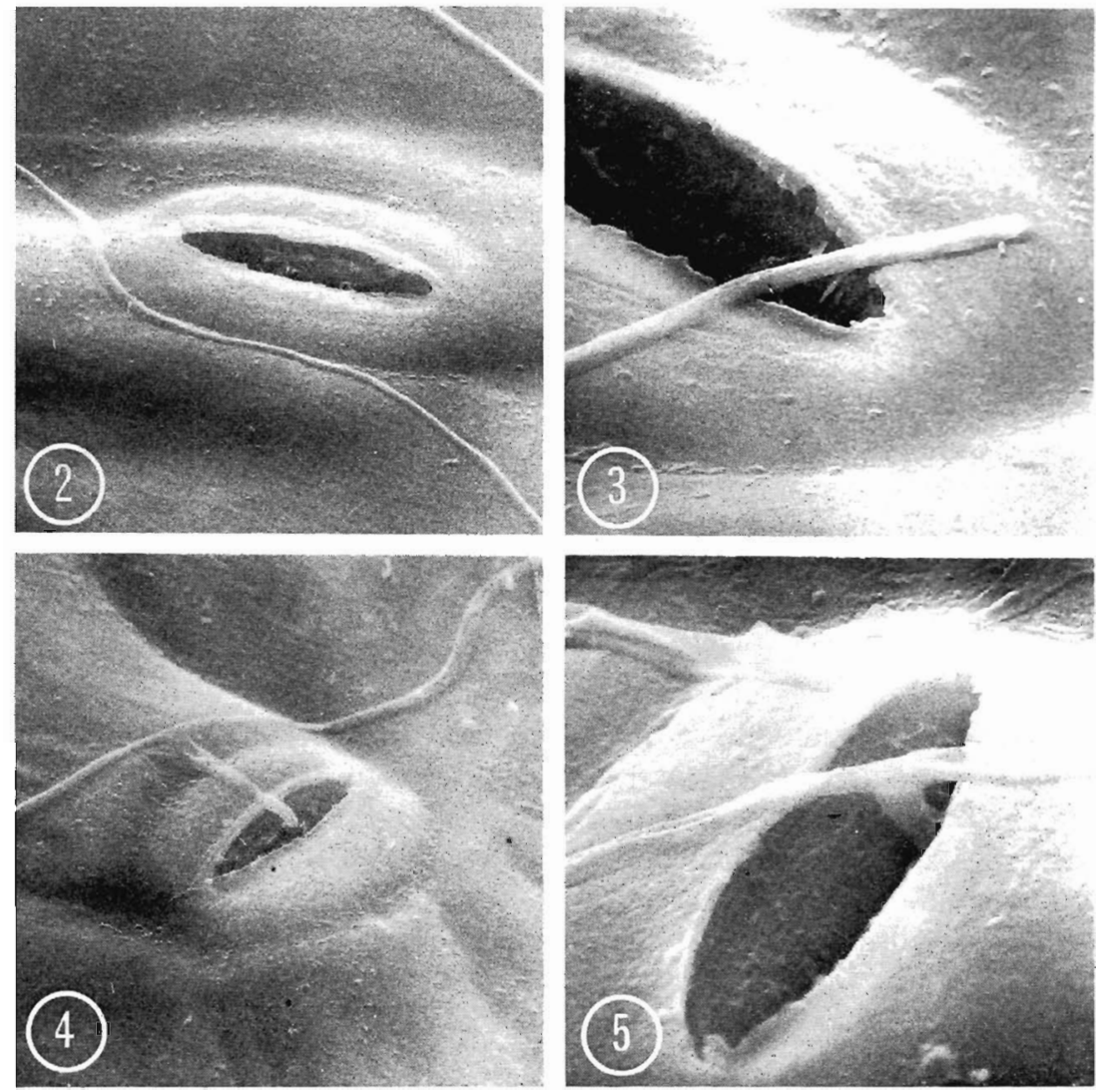

Abb. 2, 3, 4, 5. Hyphen von Cercospora beticola auf der Blattoberfläche, in der Nähe der Spaltöffnung: Die Hyphe kann an der Spaltöffnung vorbei (Abb. 2), über diese hinweg (Abb. 3) oder in diese hineinwachsen (Abb. 4, 5)

als Appressorium bezeichneten Infektionsstruktur. Sie unterscheidet sich von der Keimhyphe durch einen etwa doppelt so großen Durchmesser (Abb.6).

Nach Aufhellung mit Lactophenol und Anfärbung mit Anilinblau heben sich die dickeren Infektionshyphen im Lichtmikroskop durch ihre dunkelblaue Farbe deutlich ab. Die Konidien und die relativ dünnen Primär- und Sekundärhyphen erscheinen dagegen hellgefärbt. Dabei konnte auf der Blattoberfläche bei einzelnen Hyphen auch die Bildung von Infektionsstrukturen beobachtet werden, ohne daß diese vorher in Kontakt mit einer Spaltöffnung gekommen waren.

Nach Inkubation ohne zwischengeschaltete Trockenperiode wurden häufiger auch Penetrationen von Primär- und Sekundärhyphen ohne deutlich ausgeprägte Infektionsstrukturen beobachtet (Abb. 7). Unsere Beobachtungen über den ersten Abschnitt des Infektionsverlaufes stehen im Einklang mit denen von Pool und McKay (1916), Schmidt (1928), Darpoux et al. (1953), Canova (1959) und Solel und Minz (1971). 
Abb. 6. Infektionsstruktur einer Hyphe, die in die Spaltöffnung hinein wächst
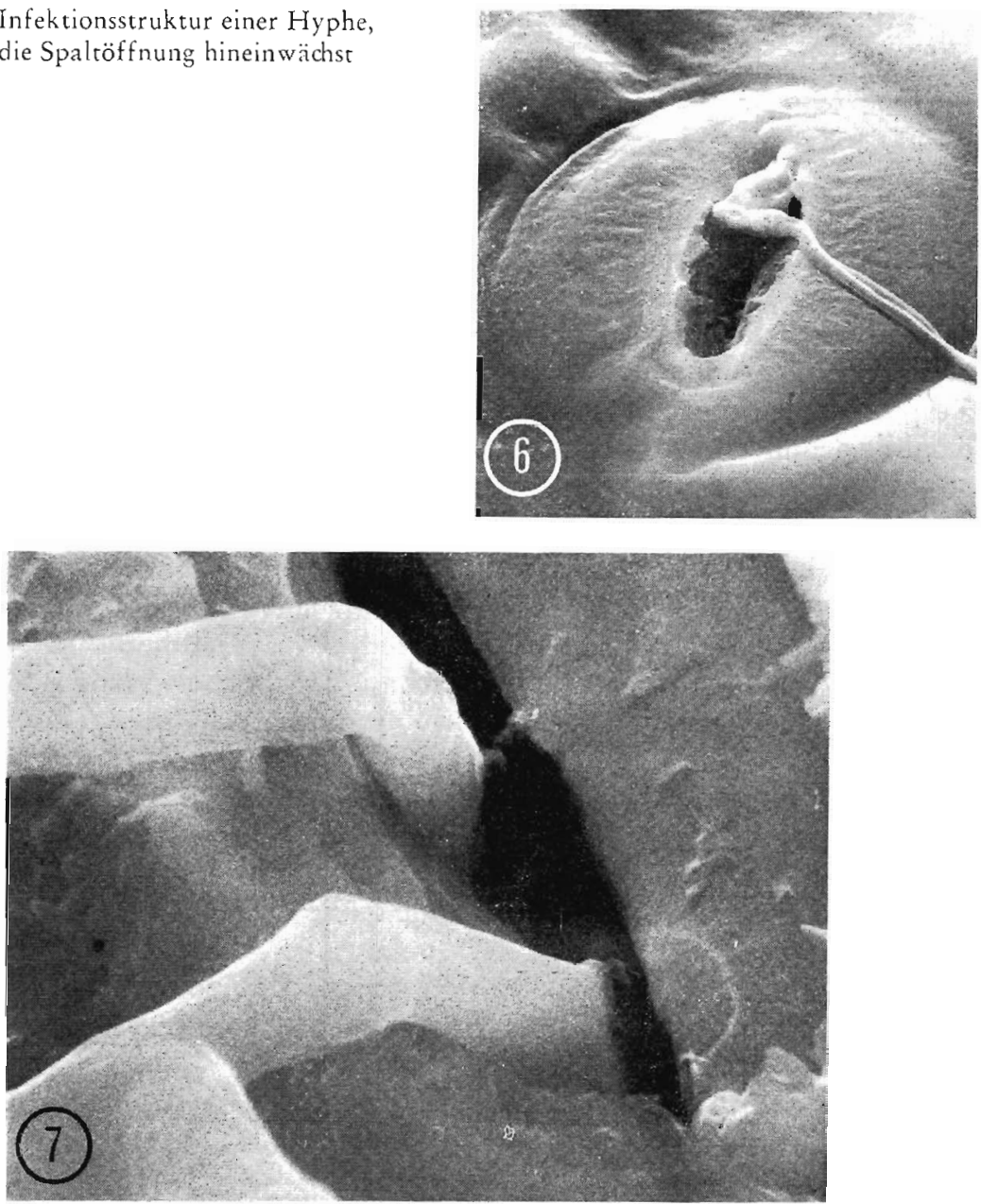

Abb. 7. Penetration von Sekundärhyphen in die Spaltöffnung, ohne Ausbildung von Infektionsstrukturen

\section{Einfluß des Blattalters auf Keimung, Hyphenwachstum und Penetration des Pilzes}

Solel et al. (1971) beobachteten eine geringere Penetrationsrate auf resistenten, zehn Monate alten Rüben im Vergleich zu anfälligen.

Dieser Hinweis auf einen Zusammenhang zwischen der unterschiedlichen Konidienkeimung und Penetration mit der Resistenz wurde von uns erneut aufgegriffen. Wir untersuchten im Gewächshaus die Konidienkeimung, das Hyphenwachstum, die Häufigkeit eines erfolgten Stomakontaktes und ermittelten die Penetrationsrate auf künstlich infizierten Blättern acht bis zehn Wochen alter Rübenpflanzen.

In einigen Fällen beobachteten wir eine verzögerte Konidienkeimung und eine geringere Penetrationsrate auf den mittleren Blättern der resistenten 
Rübenlinie im Vergleich zur anfälligen Linie. Diese Unterschiede waren aber nicht signifikant und wurden schon innerhalb einer Pflanze durch den Einfluß des unterschiedlichen Blattalters überdeckt.

Die Konidien keimten auf den älteren Blättern langsamer als auf den jüngeren. 2 d.p.i. war auf den jüngeren Blättern (viertes Blattpaar) durchschnittlich eine Konidienzelle je Konidie mehr ausgekeimt, als auf den älteren Blättern (erstes Blattpaar) (Abb. 8 A).

Gleichzeitig konnte ein verringertes Hyphenwachstum beobachtet werden, das indirekt über die Anzahl gebildeter Sekundärhyphen bzw. Hyphenverzweigungen bestimmt wurde. Im Vergleich zu den älteren Blättern hatten sich 5 d.p.i. auf den jüngeren Blättern mehr als doppelt soviel Sekundärhyphen je Konidie gebildet (Abb. 8 B).

Wenn eine Hyphe bei ihrem Wachstum auf der Blattoberfläche mit Schließzellen in Kontakt kam, wurde dies als "Stomakontakt" gewertet. Die
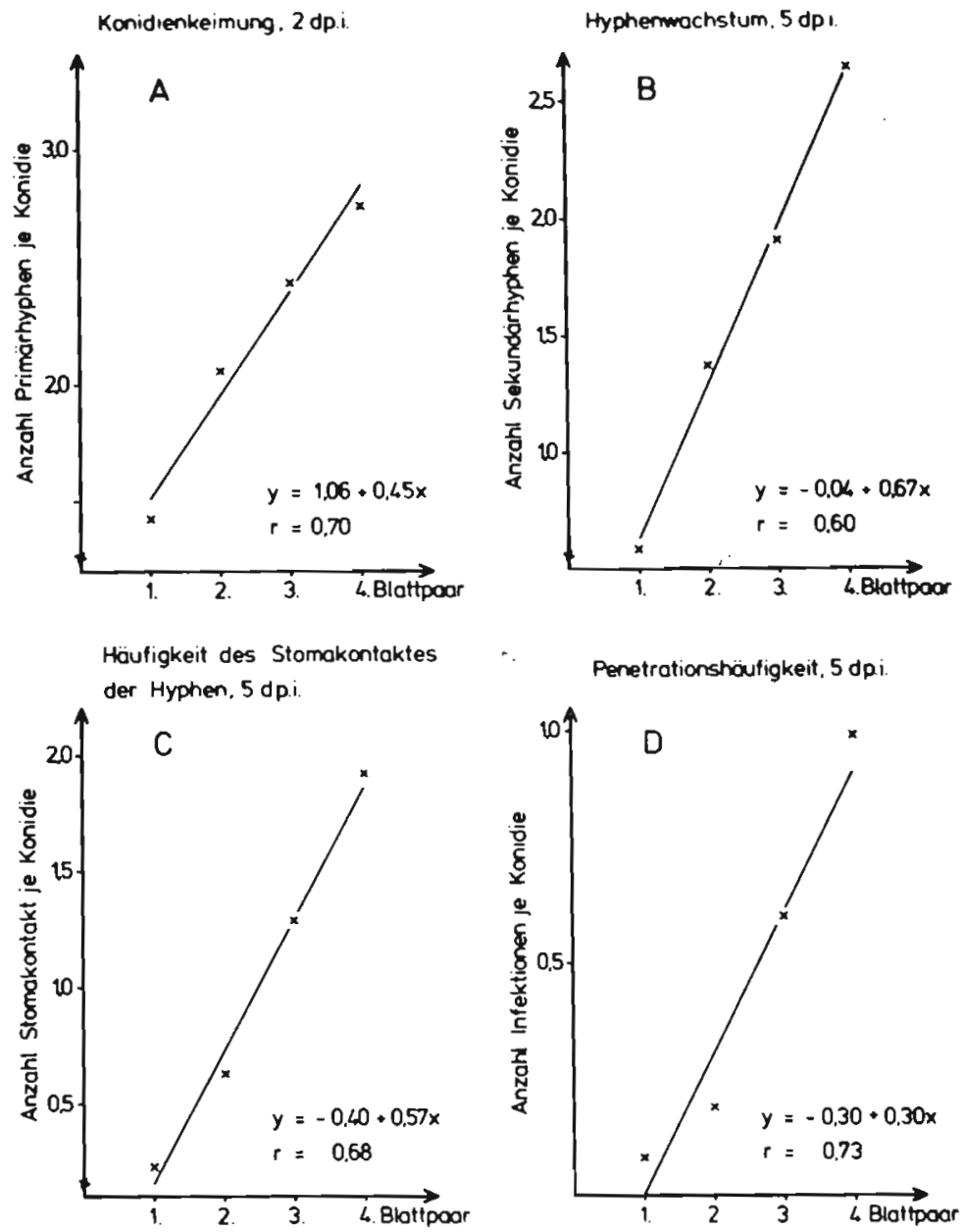

Abb. 8. Einfluß des Blattalters auf Konidienkeimung (A), Hyphenwachstum (B), Häufigkeit des Stomatakontaktes der Hyphen (C) und die Penetrationshäufigkeit (D) 
Bedeutung der unmittelbaren Nähe zur Spaltöffnung hinsichtlich eines positiv wirkenden Hydrotropismus, wird von Rathaiay (1977) erwähnt und ist ebenfalls in den Abbildungen 4 und 5 erkennbar. 5 d.p.i. waren die Hyphen einer Konidie auf den jüngeren Blättern bereits mit zwei Spaltöffnungen in Kontakt gekommen (siehe Abb. 8C). Aus jedem zweiten Stomakontakt war auf den jüngeren Blättern bereits am 5. d.p.i. eine Penetration durch die Spaltöffnung erfolgt (siehe Abb. 8D).

Die verzögerte Konidienkeimung, das verringerte Hyphenwachstum und der verminderte Stomakontakt auf den älteren Blättern könnten einige der Gründe für die hier geringere Penetrationsrate sein (siehe Abb. 8 A, B, C, D) und dadurch zu dem geringeren Befall der älteren Blätter beitragen.

\section{Die Bedeutung geöffneter Stomata für die Infektion}

Wie die vorhergehenden Ergebnisse zeigten, haben die Spaltöffnungen für den Beginn der Pathogenese eine entscheidende Bedeutung. Pool und McKaY (1916) schlossen aus ihren Beobachtungen, daß der Pilz nur durch geöffnete Stomata in das Blatt eindringen kann. Diese Vermutung wird nicht von uns, jedoch von anderen Autoren bestritten (Ruppel 1972, Rathaiah 1976). RupPEL (1972) ist der Auffassung, daß die Spaltweite für den Penetrationsvorgang der relativ dünnen Cercospora-Hyphen $(\phi=1,3$ bis $2,1 \mu \mathrm{m})$ von untergeordneter Bedeutung ist. Wir haben versucht, dies durch Beeinflussung der Offnungsweiten der Stomata zu untersuchen. Der Offnungsgrad der Stomata ist von verschiedenen Faktoren abhängig. Die Schließzellenbewegungen werden u. a. beeinflußt durch: Wasser, Licht, Temperatur, $\mathrm{CO}_{2}$-Gehalt und hormonal durch Abscisinsäure (Raschke 1975). Deshalb lag eine Anwendung dieses Phytohormons nahe.

\subsection{Einfluß von Abscisinsäure auf Spaltöffnungsweite und Infektion}

Abscisinsäure (ABS) bewirkt innerhalb weniger Minuten ein Schließen der Stomata (Mittelheuser et al. 1969) und schien deshalb für die Beantwortung unserer Fragestellung besonders günstig zu sein. An sechs bis acht Wochen alten Pflanzen applizierten wir mit einem Laborsprüher eine $40 \mathrm{ppm}$ ABS-Lösung auf die Unterseite der Rübenblätter, während die Blattoberseite, wie in allen anderen Versuchen, mit der Sporensuspension inokuliert wurde. Um den Einfluß des Blattalters auszuschalten, diente jeweils das gegenüberstehende Blatt eines Blattpaares als Kontrolle.

Messungen des Diffusionswiderstandes mit dem Diffusionsporometer zeigten $1 \mathrm{~h}$ nach Applikation einen stärkeren Spaltenschluß (= höherer Diffusionswiderstand) bei den behandelten Blättern sowohl auf der Unterseite, als auch auf der Oberseite an (Abb.9). Die Wirksamkeit der ABS war damit bewiesen. Auch nach $24 \mathrm{~h}$ war der Einfluß der ABS noch erkennbar, wobei dieser sich auf der direkt behandelten Blattunterseite stärker bemerkbar machte als bei den Spaltöffnungen der Blattoberseite (Abb. 9).

Um einen Einfluß der ABS auf den Pilz selbst ausschließen zu können, untersuchten wir die Konidienkeimung, das Hyphenwachstum und das Mycel- 


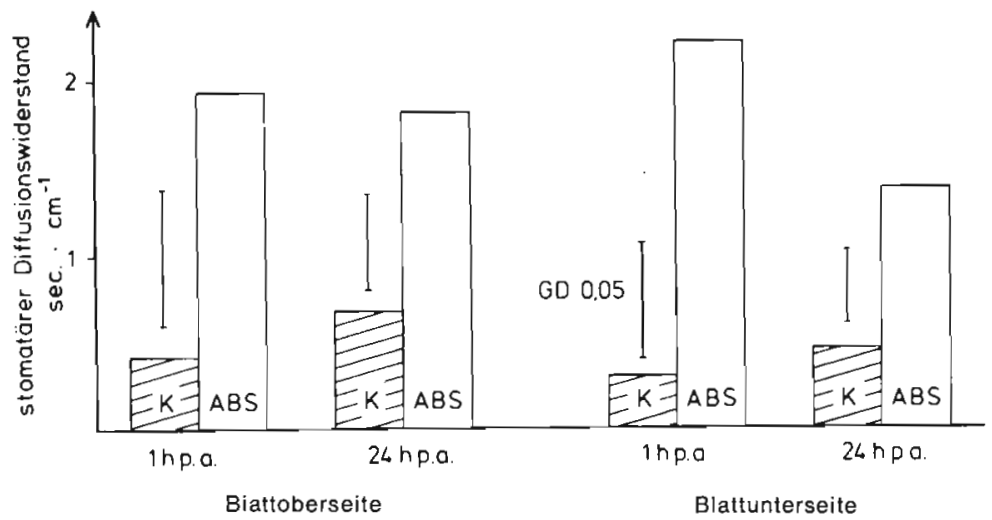

Abb. 9. Einfluß von ABS auf den stomatären Diffusionswiderstand

wachstum in vitro bis zu einer Konzentration von 300 ppm ABS. Die Auswertung der Konidienkeimung und des Hyphenwachstums auf dem Nährboden und der Blattoberfläche erbrachte keinen Unterschied zwischen ABSBehandlung und Kontrolle. Selbst nach acht Tagen konnte kein Einfluß der ABS auf das Mycelwachstum festgestellt werden. Im Infektionsverhalten des Pilzes war jedoch auf den mit ABS behandelten Blättern ein deutlicher Unterschied zu beobachten. Die Anzahl der Infektionsstrukturen und die Penetrationsrate war 5 d.p.i. stark vermindert (Abb.10 A und B). Auf den unbehandelten Blättern hatte sich im Durchschnitt eine Infektionsstruktur je Konidie gebildet. Auf den ABS-behandelten Blättern war dagegen nur bei jeder fünften Konidie eine Infektionshyphe entstanden (siehe Abb.10A). Zum gleichen Zeitpunkt war der Unterschied in der Penetrationsrate noch größer.

Die verminderte Penetrationsrate scheint dann auch einen verringerten Befallsgrad zu bedingen. Der Befallsgrad der behandelten Blätter war deutlich

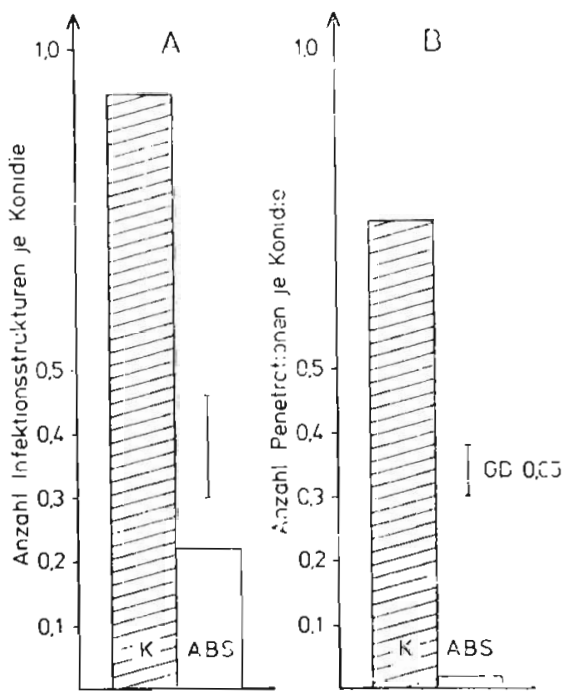

Abb. 10. Einfluß von ABS auf die Anzahl gebildeter Infektionsstrukturen (A) und erfolgter Penetration (B), 5 d.p.i. 
geringer, wenn die ABS vor dem Eindringen des Pilzes ins Blattgewebe appliziert wurde (Abb.11A und B). Wurde die ABS aber erst vom 5. bis 9. d.p.i. auf das Blatt gesprüht, also wenn bereits die meisten Penetrationen stattgefunden hatten, konnte kein Unterschied im Befall im Vergleich zur Kontrolle festgestellt werden (Abb.11C). Die Applikation von ABS vom fünften zum ersten Tag vor der Inokulation (d.p.i.) bewirkte offensichtlich noch mehrere Tage danach ein verringertes Offnen der Stomata, welches dann zu einem geringeren Befall dieser Blätter führte (Abb.11A).

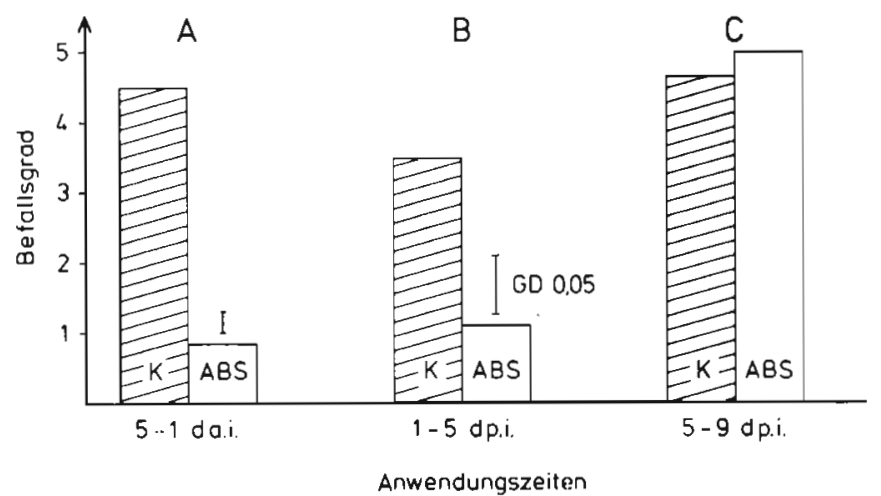

Abb. 11. Einfluß von ABS auf den Befallsgrad (18 d.p.i.) bei verschiedenen Anwendungszeiten

Auch auf den mit ABS behandelten Blättern konnten vereinzelte Nekrosen gefunden werden. Diese zeigten aber in ihrer Form, Farbe und Größe keinen Unterschied zu denen auf den Kontrollblättern. Ein direkter oder indirek ter Einfluß der ABS auf das Pilzwachstum im Blattgewebe kann damit $z$ war nicht ausgeschlossen werden, ist aber wenig wahrscheinlich.

\section{Diskussion}

Anders als z. B. Colletotrichum graminicola (PoLITIs 1976) oder Botrytis cinerea (McKeEn 1974) u. a. ist C. beticola nicht in der Lage, die Cuticula und Epidermiszellwand direkt zu durchdringen. Vielmehr sind die Cercospora-Hyphen darauf angewiesen, bei ihrem Wachstum auf der Blattoberfläche eine geöffnete Spaltöffnung zu finden, um dort eindringen zu können.

Das Hyphenwachstum auf der Blattoberfläche kann durch physikalische oder chemische Reize beeinflußt werden. Im Gegensatz zu den Verhältnissen bei verschiedenen Rostpilzen (WYNN 1976), bei denen in bezug auf die Oberflächenstrukturen des Blattes ein gerichtetes Wachstum der Keimschläuche erfolgt, konnten bei C. beticola derartige Einflüsse nicht beobachtet werden. Möglich wäre dagegen eine Wirkung der in den Blattexsudaten der Pflanze vorkommenden Substanzen, wie Kohlenhydrate, Aminosäuren, organische Säuren u. a. (Morgan et al. 1964).

Kovacs (1955) fand eine Keim- und Wachstumshemmung bei C. beticola in gesammelten Tau und Regenwasser von Rübenblättern im Vergleich zu 
destilliertem Wasser. Der hemmende Einfluß war bei den anfälligen Rübensorten geringer, bei den resistenten Sorten dagegen stärker ausgeprägt. HARrIson et al. (1961) konnten nachweisen, daß „Waschwasser" von Rübenblättern eine phenolische Komponente, Dopamin (3-Hydroxytyramin), enthält, die das Pilzwachstum hemmt. Dopamin könnte somit als präformierte fungitoxische Substanz u. a. für die stärkere Wachstumshemmung der Hyphen auf resistenten oder auch auf älteren Rübenblättern verantwortlich sein. Gegen Dopamin als alleinigen Resistenzfaktor, sowohl auf der Blattoberfläche als auch im Blattinnern, spricht allerdings ein relativ hoher Dopamingehalt einiger anfälliger Rübensorten (HARRISON et al. 1970).

SCHNEIDER et al. (1975) konnten sogar eine Förderung des Hyphenwachstums von Cercospora canescens in Blattdiffusaten anfälliger Pflanzen von Vigna unguiculata im Vergleich zu destilliertem Wasser nachweisen. Eine Wachstumsminderung stellten sie dagegen in Diffusaten resistenter Pflanzen von Vigna unguiculata fest. Die Wachstumshemmung konnte durch Zugabe von Nährlösung vermindert, aber nicht gänzlich aufgehoben werden.

Es scheint somit für Cercospora-Hyphen sowohl eine Wachstumshemmung z. B. durch fungitoxische Stoffe (Kovacs 1955, Harrison et al. 1961, HARRISON et al. 1970), als auch eine Wachstumsförderung z. B. durch ein unterschiedliches Substratangebot auf der Blattoberfläche möglich (SCHNEIDER 1975). Sortenspezifische Unterschiede in den Blattexsudaten könnten dadurch auch das Ausmaß der Penetration beeinflussen.

Das in unseren Versuchen beobachtete bessere Hyphenwachstum auf der Oberfläche jüngerer Rübenblätter im Vergleich zu älteren könnte deshalb einerseits auf einem höheren Substratangebor beruhen, das durch einen höheren Gesamtzuckergehalt jüngerer Rübenblätter (Suzuki und MorTimer 1973) verursacht wird und/oder andererseits von einer besseren Substratzugänglichkeit beeinflußt werden, die auf eine noch nicht abgeschlossene Cutinisierung und Suberinisierung der Epidermiszellwand zurückgeht (LüTTGE 1973). Eine zusätzliche Hemmung durch andere Substanzen auf älteren Blättern oder durch spezifische Inhibitoren auf Blättern resistenter Sorten ist jedoch nicht auszuschließen.

Der in unseren Versuchen festgestellte, vom Blattalter abhängige Befallsunterschied könnte eine gewisse Bedeutung für den Resistenzunterschied einzelner Rübensorten haben. Bei Sorten mit einem schnelleren Blattbildungsvermögen erreichen die Blätter ein geringeres Alter. Das bedeutet, daß sich mit einer schnelleren Seneszens der Blätter der Anteil der physiologisch jüngeren Blätter je Pflanze erhöht und dadurch zu einer unspezifischen, schnelleren Krankheitsentwicklung bei anfälligen Rübensorten beitragen könnte.

Nach der Sporenkeimung bedarf es häufig erst der Bildung einer spezialisierten Hyphenstruktur, bevor eine Penetration in den Wirt erfolgen kann (Allen 1976). Die Bildung von Infektionsstrukturen kann wirtsspezifisch von verschiedenen chemischen Stimulantien oder mechanisch von der Oberflächenstruktur ausgelöst werden (ALLEN 1976). WYNN (1976) zeigte beim Bohnenrost (U. phaseoli), daß die Strukturen der Spaltöffnung auf einem Kollodiumabdruck die Appressorienbildung induzieren können. 
Da wir bei C. beticola Hyphenwachstum über die Stomata hinweg ohne Penetration oder Bildung einer Infektionsstruktur beobachteten, dürfte eine Orientierung der Cercospora-Hyphen nach der Oberflächenstruktur auszuschließen sein. Hyphenabzweigungen unmittelbar neben oder über Spaltöffnungen lassen vielmehr eine Reaktion der Hyphen auf einen Reiz vermuten, welcher erst beim Offnen der Stomata wirksam wird.

Vor der eigentlichen Penetration schwillt die Hyphe in den meisten Fällen zu einem sogenannten "Protoappressorium" (EMmeTt und PARBery 1975) an. Dieses ist selten durch ein Septum vom übrigen Hyphenabschnitt getrennt. Die Bildung eines Appressoriums (identisch mit Infektionsstruktur) bzw. einer Infektionshyphe, wie Canova (1959) sie bezeichnet, scheint bei C. beticola u. a. von äußeren Faktoren beeinflußt zu werden.

Nach Inkubation mit nächtlichen Feuchteperioden beobachtete RathaiaH (1976) bei $99 \%$ der Infektionsvorgänge eine Appressorienbildung. Erfolgte die Inkubation bei hoher Luftfeuchte dagegen während des Tages, infizierten $82 \%$ der Hyphen ohne Bildung einer Infektionsstruktur. Bei kontinuierlicher Feuchtperiode (Tag und Nacht) hatten 50\% Penetrationen mit und 50\% ohne Appressorium stattgefunden. Rathatah schließt daraus, daß C. beticola in der Lage ist, durch geschlossene Stomata einzudringen, wenn vorher ein Appressorium gebildet wird.

Unsere Versuche mit Abscisinsäure (ABS), in denen bei Spaltenschluß der Stomata nach ABS-Behandlung eine Reduzierung der Bildung von Infektionsstrukturen und eine geringere Penetrationsrate gefunden wurden, sprechen jedoch nicht für diese Annahme. Im Gegenteil, wir konnten ein Eindringen der Hyphen nur bei geöffneten Stomata beobachten. Die $82 \%$ direkten Penetrationen ohne Appressorium während der "kontinuierlichen "Feuchtperiode in Rathaiah's Versuchen lassen unserer Ansicht nach vielmehr den Schluß zu, daß geöffnete Stomata bei ausreichend hoher Luftfeuchtigkeit entweder keinen Reiz auslösen oder, daß unter diesen Bedingungen keine Notwendigkeit für die Bildung von Infektionsstrukturen vor der Penetration besteht. Die Bildung von Appressorien im Dunkeln könnte dagegen eine Ansammlung von Cytoplasma im Hyphenschlauch und damit ein Anschwellen über den des nachts nicht ganz geschlossenen Spaltöffnungen (CANOva 1959) sein, die zwar auf Grund ihrer geringen Spaltenweite ein Eindringen nicht mehr ermöglichen, aber trotzdem noch einen Reiz wirksam werden lassen und damit die Bildung der Infektionsstruktur induzieren.

Die Penetration erfolgt dann anschließend am Tage bei größeren Spaltweiten und ist auf Grund der stomatären Transpiration und des "Randcffektes" der Stomata (Hess 1974) wahrscheinlich auch bei geringerer äußerer relativer Luftfeuchte noch möglich.

In einer neueren Arbeit ist Rathaiah (1977) ebenfalls der Ansicht, daß unterschiedliche Feuchtegradienten auf der Blattoberfläche in unmittelbarer Vähe der Stomata die Penetrationsrate des Pilzes beeinflussen.

Ruppel (1971) konnte keine Korrelation zwischen Stomadichte und Resistenzgrad verschiedener Rübensorten feststellen. Der vermehrte Stomakontakt der Hyphen, und die damit verbundene höhere Penetrationsrate, die wir in 
unseren Versuchen auf den jüngeren Blättern im Vergleich zu den älteren fanden, dürfte unserer Ansicht nach zu einem größeren Teil auf das verbesserte Hyphenwachstum und nur zu einem geringeren Teil auf die höhere Stomadichte dieser Blätter zurückzuführen sein.

Der Infektionserfolg von C. beticola auf $B$. vulgaris wird zwar einerseits von den Faktoren wie 1. Hyphenwachstum auf der Blattoberfläche, 2. Stomadichte und Häufigkeit des Stomakontaktes und 3. der Offnungsweite der Stomata stark beeinflußt, die wiederum in engem Zusammenhang zum Blattalter stehen; andererseits scheinen diese Faktoren aber nur einen Teil der Resistenzmechanismen abzudecken, welche die unterschiedliche Anfälligkeit verschiedener Rübensorten erklären könnten. Der Einfluß des Blattalters deutet schon darauf hin, daß weitere Resistenzmechanismen im physiologischen Bereich der Wirt-Parasit-Reaktionen zu suchen sind. Vor entsprechenden stoffwechselphysiologischen Untersuchungen erschien es uns daher sinnvoll, in einer weiteren Arbeit zunächst das Verhalten der Hyphen im Blattgewebe zu verfolgen, um den Zeitpunkt des Einsetzens der Abwehrreaktionen der Pflanze im Verlauf der Pathogenese zu erfassen (Feindt, Mendgen und Heitefuss 1981).

\section{Zusammenfassung}

Aus den Infektionsversuchen in Klimakammer und Gewächshaus mit Cercospora beticola auf Zuckerrübe (Beta vulgaris) unterschiedlicher Anfälligkeit und Resistenz geht hervor, daß die geringere Anfälligkeit der resistenten Rüben sich erst mit zunehmendem Pflanzenalter manifestiert. Erst bei sieben bis acht Wochen alten oder älteren Pflanzen konnte die Unterscheidung zwischen "anfällig" und "resistent" gemacht werden.

Latenzzeit und Befallsgrad zeigten eine deurliche Abhängigkeit vom Blattalter. Jüngere Blätter resistenter Pflanzen erreichten den gleichen Befallsgrad wie ältere Blätter anfälliger Pflanzen.

Geringere Unterschiede in Konidienkeimung, Hyphenwachstum und Penetrationsrate waren, unabhängig vom Resistenzgrad der beiden Sorten, auf Blättern gleichen Alters nicht signifikant. Innerhalb einer Pflanze waren allerdings alle drei Parameter auf den älteren Blättern im Vergleich zu den jüngeren signifikant vermindert.

Versuche mit Abscisinsäure (ABS) deuten darauf hin, daß CercosporaHyphen nur durch geöffnete Stomata eindringen. Der Befallsgrad war stark reduziert, wenn die Blätter vor der Penetration des Pilzes mit ABS in nicht fungitoxischen Konzentrationen behandelt worden waren.

\section{Summary}

The influence of stomatal opening and leaf age on the infection by Cercospora beticola in resistant and susceptible leaves of Beta valgaris

Plants of a resistant and a susceptible sugar beet (Beta vulgaris) cultivar were inoculated with a spore suspension of Cercospora beticola to examine the 
infection process and disease severity under certain growth conditions in relacion to host and leaf age. The results obtained in a grow th chamber and/or green house indicated, that the lower susceptibility of the resistant cultivar becomes evident only with increasing plant age. Only with plants $7-8$ weeks old or more the differentiation between susceptible and resistant ones could be made under the given growth conditions.

When single leaves were compared, fungal attack was severe on young leaves but less on older leaves, independent of susceptibility, i.e. disease severity was similar on young leaves of resistant plants compared to old leaves of susceptible plants.

Spore germination, hyphal growth and penetration through stomata were similar on leaves of the same age of either the resistant or the susceptible cultivars, but were reduced on old leaves when compared with young leaves of both cultivars.

Experiments with abscisic acid (ABA) indicated that penetration of the Cercospora hyphae occurs only through open stomata. Treatment with nonfungicidal concentrations of $A B A$ reduced disease severity if applied to the leaf before penetration of the hyphae.

Die Arbeit wurde aus Mitteln der GFP (Gesellschaft zur Förderung der privaten deutschen Pflanzenzüchtung) gefördert.

Herrn Dipl.phys. R. Strey, Max-Planck-Institut für Biophysikalische Chemie in Göttingen, danken wir für die Möglichkeit zur Durchführung der Untersuchungen mit dem Rasterelektronenmikroskop.

\section{Literaturverzeichnis}

AlleN, P. J., 1976: Control of spore germination and infection structure formation in the fungi. In: R. Heitefuss and P. H. Williams (Eds.), Encyclopedia of Plant Physiology, Vol. 4 : Physiological Plant Pathology, 51-85. Springer-Verlag, Berlin.

Blerholder, H., 1971: Beiträge zur Epidemiologie von Cercospora beticola Sacc. an Zuckerrübe. Diss. Bonn, Landw. Fak.

Boyde, A., and C. Wood, 1969: Preparation of animal tissues for surface-scanning electron microscopy. J. Microscopy $90,221-249$.

Brillova, D., O. Sladka, and A. Michalikova, 1973: Defensive reactions of the sugar beet after the infection with Cercospora beticola Sacc. Biologia (Prace) 19, 1-65.

Canova, A., 1959: Richerche su la biologia e l'epidemiologia della Cercospora beticola Sacc. An. Sperim. Agr. (Roma) 13,1-251.

Darpoux, H., A. Lebrun, et M. Arnoux, 1953: Sur le phénoméne de la contamination par le Cercospora beticola. Phytiatrie-Phytopharmacie 4, 125-144.

Day, P. R., and K. J. ScotT, 1973: Scanning electron microscopy of fresh material of Erysiphe graminis f. sp. hordei. Physiol. Plant Path. 3, 433-435.

Emmett, R. W., and D. G. Parbery, 1975: Appressoria. Ann. Rev. Phytopath. 13, 147-167.

FEINDT, F., 1977: Untersuchungen zum Infektionsvorgang von Cercospora beticola Sacc. auf Beta vulgaris L. bei unterschiedlicher Anfälligkeit. Diss. Görtingen.

- - K. Mendgen und R. Heitefuss, 1981: Feinstruktur unterschiedlicher Zellwandreaktionen im Blattparenchym anfälliger und resistenter Rüben (Beta vulgaris L.) nach Infektion durch Cercospora beticola Sacc. Phytoparh. Z. (im Druck).

FrasDSEN, N. O., 1956: Untersuchungen über Cercospora beticola. IV-V. Zucker 9, 3-5, $51-53$.

Gerlach, D, 1969: Botanische Mikrotechnik. Georg Thieme Verlag, Stuttgart. 
Harrison, M., G. W. Maag, R. J. Hecker, and M. G. Payne, 1970: Some speculations on the role of dopamine on the resistence of sugar beets to Cercospora leaf spot. J. Amer. Soc. Sugar Beet Techn. 16, 34-40.

- -, M. G. Payne, and J. O. Gaskill, 1961 : Some chemical aspects of resistance to Cercospora leaf spot in sugar beets. J. Amer. Soc. Sugar Beet Techn. 11, 457-468.

Hecker, R. J., E. G. Ruppel, G. W. MaAG, and D. M. Rasmuson, 1975: Amino acids associated with Cercospora leaf spot resistance in sugar beet. Phytopath. Z. 82, 175-181.

Hess, D., 1974 : Pflanzenphysiologie, 3. Aufl. Ulmer Verlag, Basel/Stuttgart.

Jchnson, G., D. D. MaAg, D. K. Johnson, and R. D. Thomas, 1976: The possible role of phytoalexins in the resistance of sugarbeet (Beta vulgaris) to Cercospora beticola. Physiol. Plant Path. 8, 225-230.

Kanemasu, E. T., G. W. Thurtell, and C. B. Tanner, 1969: Design, calibration and field use of a stomatal diffusion porometer. Plant Physiol. 44, 881-885.

Kovács, A., 1955: Über die Ursachen der unterschiedlichen Resistenz der Zuckerrübensorten gegen Cercospora beticola Sacc. Phytopath. Z. 24, 283-298.

LA, Y. J., 1963: Conidial production of Cercospora beticola Sacc. on tomato juice agar. Korean J. Bot. 6, 8-10.

Lüttge, U., 1973: Stoffrransport der Pflanzen. Springer-Verlag, Berlin.

Martin, S. S., 1977: Accumulation of the flavonoids betagarin and betavulgarin in Beta vulgaris infected by the fungus Cercospora beticola. Physiol. Plant Path. 11, 297-303.

MCKEEN, W. E., 1974: Mode of penetration of epidermal cell walls of Vicia faba by Botrytis cinerea. Phy topathology 64, 461-467.

Mixtelmeuser, C. J., and R. F. M. van Steveninck, 1969: Stomatal closure and inhibition of transpiration induced by (Rs)-abscisic acid. Nature 221, 281-282.

Morgan, J. V., and H. B. Tukey, 1964: Characterization of leachate from plant foliage. Plant Physiol. 39, 590-593.

PoLiris, D. J., 1976: Ultrastructure of penetration by Colletotrichum graminicola of highly resistant oat leaves. Physiol. Plant Path. 8, 117-122.

Pool, V. W., and M. B. McKay, 1916: Relation of stomatal movement to infection by Cercospora beticola. J. Agric. Res. 22, 1011-1038.

RAschKe, K., 1958: Über den Einfluß der Diffusionswiderstände auf die Transpiration und die Temperatur eines Blattes. Flora 146, 546-578.

- -, 1975: Stomatal action. Ann. Rev. Plant Physiol. 26, 13-29.

Rathaiah, Y., 1976: Infection of sugarbeet by Cercospora beticola in relation to stomatal condition. Phytopathology 66, 737-740.

- - 1977: Stomatal tropism of Cercospora beticola in sugarbeet. Phytopathology 67, $358-362$.

Ruppel, E. G., 1972: Variation among isolates of Cercospora beticola from sugar beet. Phytopathology 62, 134-136.

- -, 1972: Negative relationship of stomatal size and density with resistance in sugar beet to Cercospora beticola. Phytopathology 62, 1095-1096.

SAMPSON, J., 1961: A method of replicating dry or moist surfaces for examination by light microscopy. Nature 191, 932-933.

Schmrdr, E. W., 1928: Untersuchungen über die Cercospora-Blattfleckenkrankheit der Zuckerrübe. Arb. Zuckerfabrik Kleinwanzleben 4, 3-40.

SCHNeIDeR, R. W., and J. B. Sinclair, 1975: Inhibition of conidial germination and germ tube growth of Cercospora canescens by cowpea leaf diffusates. Phytopathology 65, $63-65$.

SOLEL, Z., and G. Mrnz, 1971: Infection process of Cercospora beticola in sugarbeet in relation to susceptibility. Phytopathology 61, 463-466.

Suzuki, M., and D. C. Mortimer, 1973: Sugar concentration gradient of the sugar beet plant in relation to translocation. Canad. J. Bot. 51, 1733-1739. 
WheEleR, H., 1977: Ultrastructure of penetration by Helminthosporium maydis. Physiol. Plant Path. 11, 171-178.

WYNN, W. K., 1976: Appressorium formation over stomates by the bean rust fungus: Response to a surface contact stimulus. Phy topathology 66, 136-146.

Zelrtch, J., 1961: Biochemical control of stomatal opening in leaves. Proc. Nat. Acad. Sci. 47, $1423-1433$.

Anschriften der Verfasser: Dr. F. FEindT, Dreisenweg 2, D-3354 Dassel-Lüthorst. Prof. K. Mendgen, Lehrstuhl für Phytopathologie der Universität Konstanz, D-7750 Konstanz. Prof. R. Heitefuss, Institut für Pflanzenpathologie der Universität Göttingen, Grisebachstr. 6, D-3400 Göttingen. 